\title{
WEIGHT SEMI HIDDEN MARKOV MODEL AND DRIVING SITUATION CLASSIFICATION FOR DRIVER BEHAVIOR DIAGNOSTIC
}

\author{
Nicolas Dapzol \\ LESCOT / INRETS \\ Lyon / France \\ E-mail: dapzol@inrets.fr
}

\begin{abstract}
Summary: In this study, we propose to use statistical modelling to analyze, model, and categorize driving activity. To achieve this objective, we develop a new statistical model by adding a weight feature to the classic Semi Hidden Markov Model (SHMM) framework. Then, to assess its capacity, we conduct an experiment that allows us to record 718 driving sequences categorized in 36 situations. We then used our modelling to identify the driver's aim and the driving situation he's in. Furthermore, we adapted the ascendant hierarchic classification technique to this modelling. It allows us to understand which situations are close and to define partitions of whole driving situations. Finally, on these sequences, our modelling choice allows us to predict the driver's situation with, on average, an $85 \%$ success rate. These results show the HMM effectiveness to manage temporal and multidimensional data by modelling predicting drivers' behavior.
\end{abstract}

\section{INTRODUCTION}

To increase comfort and safety in vehicles, integrated communicative systems are developed. These systems could have various objectives and interact with drivers in different ways. Navigation systems and anti-collision systems are examples of such systems.

Nevertheless, even if these systems often increase security, most of them don't take into account the driving situation when providing their assistance. Yet, trying to help the driver without knowing his specific behaviour and the specific situation he's in brings the risk of giving him inadequate assistance. This could disturb him at a critical moment. To avoid this, the interaction with the driver has to be adapted to his own behavior. Thus, the existence of a sub-system able to understand the current driving situation and to manage the interaction with the driver will increase system effectiveness.

The current driving situation analysis could now be done using the data on the vehicle dynamics and on the driver's actions collected at each moment on the vehicle via the CAN bus.

Nevertheless, in order to categorize the driver's situation, every system needs to compare a data temporal series with a library of driving situation modeling and then select the most adequate.

Unfortunately, modeling these data is complex. They are large in number, noisy, time-related and change according to the driver, the moment, and the environment characteristics. Thus, to model the data recorded during driving situations, various models have been used, such as linear system, statistical techniques or neural network.

More recently, the Hidden Markov Model (HMM) has been used to model the driving data 
evolution. Studies [Pentland \& Liu, 1999] have shown that this modeling seems adequate to understand, model and predict drivers' behavior.

Indeed, modelling applied to the driver behavior data has to process multi-dimensional and temporal data. HMM appears to be effective in that. Indeed, it integrates 3 characteristics essential for driving data analysis.

1. Existence of efficient algorithms to estimate the modelling parameters. Although some authors point out that methods based on expert rules give interesting results both in terms of prediction and behavior analysis, these methods are built upon the visual analysis of the data and the definition of relevant criteria. They required a long process of analysis and error/success (Tattegrain-Veste et al, 2004). So, due to the large amount of data and their complexity, an automatic learning process is necessary.

2. A real-time adequation criterion of new data set to a model.

In order to help the driver in a secure way, the situation he's in has to be quickly categorized. Although they study a small number of situations (6), Pentland et al. show that HMM could be used to quickly predict drivers' situations with a good recognition rate (almost 95\%). Oliver \& Pentland, and Kumagai et al., obtain almost the same results with other situations and different sensors. It shows the effectiveness of the modeling for quickly categorization (Oliver \& Pentland, 2000; Kumagai et al., 2003).

3. Modeling interpretation.

Due to the large diversification of driving situations, the record of a few sets of examples during experimentation can be biased by uncontrollable factors (weather, traffic ...). The presence of these factors could create "noise" able to change normally recorded data in these situations. Thus, when a model is built on this data, we need an expert point of view to validate /invalidate its capacity to be a general model. Indeed, in Pribe (1999), authors show that neural networks give good results. Nevertheless, as this modelling is like a "black box," understanding mistakes were difficult, and so was its improvement. Thus, in order to have this expert point of view, the modelling has to be interpretable in terms of driving activity. Kumagai et al. (2003) shows that HMM could be used to interpret the driving activity as a succession of phase. Although this interpretation is due to the simplicity of the variables used (the speed and braking), it convinced us even more of the advantage of using HMM to understand driving activity.

With these 3 qualities, HMM seems to be fit for driving data analysis. Although good results were obtained, few situations were analysed. We think that if we want to study a larger number of situations, this modeling has to be adapted to be more effective.

The second section defines the development we made both in terms of modelling and in terms of algorithms. In the third part, we present the experimentation we conducted. Finally, we will present and discuss our results. 


\section{WEIGHT SEMI HIDDEN MARKOV MODEL}

\section{Hidden Markov Model: presentation}

The Hidden Markov Model (HMM) is a stochastic signal model. It aims to model discrete or continuous multidimensional signal by a random process. This framework supposed that the signal evolution can be considered as the evolution of 2 processes. The first one is the visible signal, like what we can record from the sensors. It's said to be the visible process, named $\mathbf{Y}$. Its value depends on another process. This second process, $\mathbf{S}$, is why we can see that on the first process at each moment. Its values are not directly observable but can be derived from the analysis of $\mathbf{Y}$. This process is generally evolving state by state and fits the classic Markov constraints (Rabinner, 1989). The relation between $\mathbf{Y}$ and $\mathbf{S}$ is defined by a density function.

The model's structure allows to it process large quantities of temporal and multi-dimensional data. These qualities were first successfully used in the field of voice recognition. In this field, the invisible process was phoneme and the visible one the vocal signal. Using the vocal signal, words spoken could be precisely diagnosed. It's now used in various applications like gene research, eye tracking or, as we have seen before, driver behavior recognition.

\section{Weight Semi Hidden Markov Model}

To improve its effectiveness in this last field, we hypothesize that using driving expert knowledge to develop a specific HMM will increase modelling efficiency. Indeed, preliminary research shows that driving activity has two important particularities.

1. From a psychological point of view, the driving activity could be divided into phases (Bellet, 1998). Moreover experiences show that these phases are relatively stable. In order to integrate this important characteristic, we choose to use Semi Hidden Markov Model (Rabinner, 1989). This modelling is an extension of the HMM framework, which allows us to precisely model the time spent in each state. Moreover, we suppose that time spent in each state must be superior to 1 second.

2. Depending on the situation, some sensors may bring more information than others (Salvucci \& Liu, 2002). For example, to model a "Turn left," the steering wheel seems more important than the speed of the vehicle. So, we were led to use a weight concept on the variable. To implement this specificity, we develop a specific modeling the weight Hidden Markov Model (Dapzol, 2006). This modelling allows us to define for each situation and for each state, what variables are the most relevant.

The Weight-Semi HMM (WSHMM) was developed as extension of HMM which takes the 2 previous features. It specifically models time spent in each state and variable importance. All algorithms for learning and prediction could be found in Dapzol (2006). 


\section{EXPERIMENTATION}

To assess our model, we conduct an experiment that aims to record behaviour data on various real driving situations. We choose to study the specific effect of infrastructure and driver's aim on behavior. So, we set other factors which could influence the activity.

The 5 subjects were chosen in order to have a homogeneous population in term of age and driving experience (about 40 years old and experiment drivers). The instruction was to drive naturally (overtake if they feel it necessary; go to the speed they want...). Furthermore, in order to interfere as little as possible, the driving directions were given as soon as possible. In the same way, the duration of the experiment was about only $1 \mathrm{~h}$ (depending on traffic) in order to ensure that driver fatigue did not change behaviour. Finally, the experiment took place only when the weather was sunny and the visibility good.

Subjects had to drive a predetermined urban course. This course was situated in town and motorway and was chosen to include various driving situations (intersection and left turn, round about and straight road....). We used an experimental vehicle that allowed us to record data from the driver (clutch, accelerator, steering wheel ...), from the vehicle (speed and longitudinal acceleration), and from the environment (the front and the back scene were recorded on a video).

With this we defined driving sequences by a period where the driver has a homogeneous tactical aim and we characterized theses sequences by the urban context (town, motorway, etc.), the initial speed, the infrastructure, the aim of the driver (as categorised from an expert view), an $\mathrm{d}$ the different events which occurred in the sequence-pedestrian crossing the street, car overtaking the driver. In this way, 1209 driving sequences were defined, interpreted and characterized. The average sequence length is about 15 seconds. Of course, each sequence was associated with sensors evolution data.

Then, we defined "driving situation" as a group of sequences homogeneous in terms of urban context, infrastructure, aim of the driver, and the initial speed. Unfortunately, some external events changed the normal organization of the driver's action (activity of another vehicle, change of light when crossing an intersection, etc.). To model the driver's action, we just kept sequences without external events ( 718 sequences). To allow the constitution of a group of test sequences and of learning sequences for each situation, we kept only situations with more than 5 sequences. Thirty-six situations met this criterion (turn-left at medium speed in an X intersection; overtake at low speed in a straight line ...). In each situation, we have 19, 5 -sequences on average (see Dapzol, 2006, for more details). These data were then used to implement and test our modelling.

\section{RESULTS}

In this section, we present results obtained using our new model, and a method we developed to manage the diversity of driving situations. 


\section{Model Implementation Method and Partitions Definition}

We associated each of the 36 driving situations with a WSHMM. Using learning algorithms, we computed for each WSHMM the optimal parameter of each modelling. To optimize the number of state and the topology (the different link between states), we used topology learning algorithms and expert validation, which are presented in Dapzol (2006).

Although we optimized each model parameter, driving situations are sometimes very similar. For example, depending on the driver and on collected data, the behavior while turning at a $\mathrm{T}$ intersection and turning at an $\mathrm{X}$ intersection often seems the same. So, in order to avoid confusion, we chose to automatically group some situations and to define different partitions for all the situations. As models can be very different in terms of topology and learning sequence number, we developed a specific method.

The technique we developed is based on a probabilistic distance between situation and hierarchic ascending classification (Dapzol, 2006). We first considered every situation separately. Then we computed the distance between each of them. To compute the distance between 2 situations, we chose the pseudo-distance defined by Nechyba \& $\mathrm{Xu}$ for the HMM (Nechyba \& Xu, 1998). This distance has the advantage of evaluating the proximity between models with different dimensions by computing the model likelihood regarding the learning sequence associated to each one.

The process consisted of considering first all the modelling, then computing. When this distance is computed, the 2 closest situations are grouped.

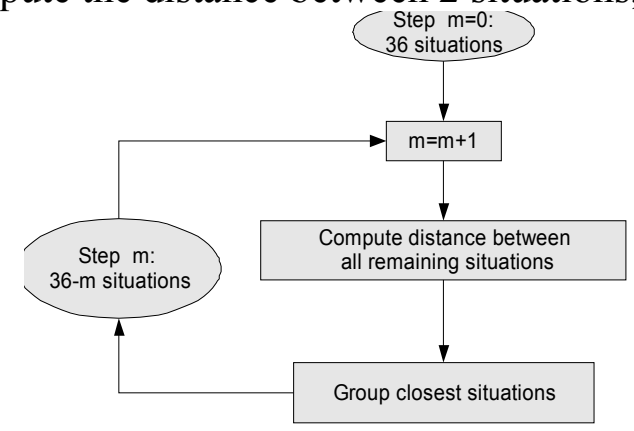

Figure 1. Hierarchic classification process to group driving situations.

Then we process iteratively. Distance between all situations is re-calculated on the new partition; and another, the 2 closest situations are grouped. This process is done until one group stayed (Figure 1).

Using this method, the total number of situations was divided into 3 different ways:

- A large partitioning composed of 26 groups of situations. The situations are mainly grouped regarding the infrastructure. Recognition rate here is $75.35 \%$.

- A medium partitioning composed of 18 groups of situations, which grouped situations with heterogeneous behaviours. Recognition rate is $87.88 \%$.

- A small partitioning composed of 12 groups of situations where the recognition rate is high $(90.53 \%)$ 


\section{Test}

We tested our modelling effectiveness on these 3 partitions using 2 criteria: "off line diagnostic" and "on line diagnostic." For each of the 592 test sequences, we computed the most probable situation using our modelling base.

To point out the real utility of our modelling, we make the same test with HMM, and Semi HMM. The first criterion is evaluated on all the data of each test sequence (Figure 2). This criterion is useful to test if the modelling was able to discriminate different pattern for each situation. In future, the possibility of using this "off line" modelling criteria could be used to label data automatically.

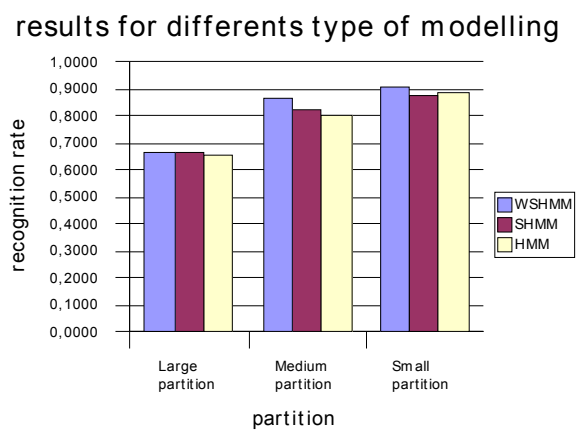

Figure 2. Recognition rate for the a posteriori criterion according to modelling type

The WSHMM seems more effective than the HMM for the small partition (91\% vs. 88\%) and a lot more for the intermediate partition ( $88 \%$ vs. $80 \%)$.

We also tested our modelling with a second criterion. The "online diagnostic" was calculated using only the $t_{0}$ first seconds $\left(t_{0}=1,2\right)$ of each sequences. This criterion is useful to evaluate the model capacity to give an earlier diagnostic.

In this case, the recognition rate is good $(75 \%$ for the intermediate partition and for recognition in 1 second). The rate "online" 2 seconds after the beginning of the sequences is close to the rate "offline" (see Figure 3).

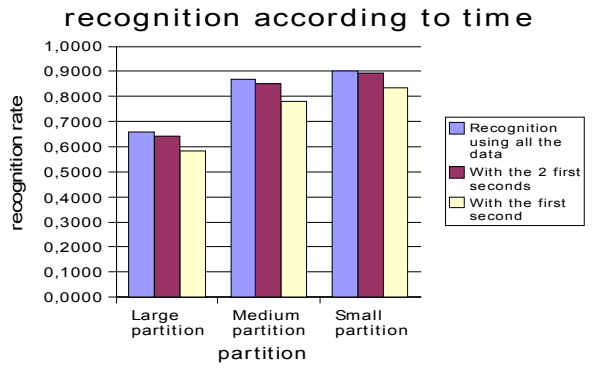

Figure 3. Recognition rate according to time using WSHMM

Moreover, confusion seems due not to a modelling problem but to a natural difficulty to discriminate some situations in the first seconds. Indeed for some situations, the simplicity of the sensors used doesn't allow us to predict the situation earlier. For others, only the analysis of the final state of the vehicle could categorize them correctly. For example, sequences in which the driver turns in an intersection and those in which the driver stops are badly recognised with only the first second's data. Only the study of eye movements could improve the recognition rate.

These results confirm our choices in our modelling. Indeed, if a small number of situations could be classified using HMM, a large number of situations brings a supplementary complexity. The integration of specific features matched with the driving activity characteristics (weight, time modelling) could be a solution to this problem. 


\section{CONCLUSION}

To manage in a secure way interaction between driver and electronic system, data collected on driver behavior has to be analysed. Nevertheless, due to their complexity, no system could categorize driving situation in a secure way. To improve previous modeling, we extend the HMM to the Weight Semi Hidden Markov Model, which allows us to model data time course explicitly and add a weight feature. Moreover, we adapt ascendant classification to this modelling.

To test our model, we conducted an experiment in real conditions, and we assessed our model using two criteria that indicated our modelling choices increased the recognition rate compared to other modelling types. We showed that with more situations analysed, our results are closed than those of previous studies.

From a practical view, the weight feature and algorithms associated could be used to find the variables necessary to discriminate situations. Yet, even if the main study considers driving as a succession of independent driving sequences, a long-term dependency between sequences exists. Integrating this characteristic will improve not only the recognition rate but also knowledge of driver behaviour.

\section{REFERENCES}

Bellet, T. ( 1998). Modélisation et simulation cognitive de l'opérateur humain: une application à la conduite automobile. Phd thesis. Université Paris V.

Dapzol, N. (2006). Analyse de l'activité de conduite par les chaînes de Markov cachées et les modèles de ruptures multiphasiques: méthodologie et applications. Phd thesis. Claude Bernard University.

Kumagaï T., Sakaguchi Y., Okuwa M. \& Akamatsu M. (2003). Prediction of driving behavior through probabilistic inference. In, Proceedings Of The Eight International Conference On Engineering Application Of Neural Networks.

Nechyba, M., \& Xu, Y. (1998). Stochastics similarity for validating human control strategy models. In, Proceedings IEEE Trans. on Robotics and Automation, 14(4), 437-451.

Pentland, A. \& Liu, A. (1999). Modeling and prediction of human behavior. Neural Computation $11,229-242$.

Pribe, C. \& Rogers, S. (1999). Learning to associate observed driver behavior with traffic controls. Transport Research Record 1679, 95-100.

Rabinner. L. (1989). A tutorial on hidden markov models and selected applications in speech recognition. In, Proceedings of the IEEE, vol 77(2), 257-286.

Salvucci, D., Liu, A. (2002). The time course of a lane change: driver control and eye-movement behavior. Transportation Research Part F 5, 123-132.

Oliver, N. \& Pentland, A. (2000). Graphical models for driver behavior recognition in a smart car. In IEEE Conference On Intelligent Vehicles, Detroit. 